\title{
Functional connectivity in a baseline resting-state network in autism
}

\author{
Vladimir L. Cherkassky, Rajesh K. Kana, Timothy A. Keller and Marcel Adam Just \\ Center for Cognitive Brain Imaging, Department of Psychology, Carnegie Mellon University, Pittsburgh, Pennsylvania, USA \\ Correspondence and requests for reprints to Dr Vladimir L. Cherkassky, PhD, Center for Cognitive Brain Imaging, Department of Psychology, \\ Carnegie Mellon University, Pittsburgh, PA 15213, USA \\ Tel: + I 412268 3379; fax: + I 412268 2804; e-mail: cherkassky@cmu.edu
}

Acknowledgements: This research was supported by the Collaborative Program of Excellence in Autism (CPEA) Grant HD35469 from the

National Institute of Child Health and Human Development.

Received 26 July 2006; accepted 28 July 2006

Brain activity in people with high-functioning autism has been shown to be atypical in a number of ways, including reduced synchronization across areas of activation measured by functional magnetic resonance imaging. This activation atypicality has been observed mostly during the performance of cognitive tasks. This study compares the resting-state network of 57 participants with autism and 57 control participants matched for age and intelligence quotient. The results indicate that both groups have a resting-state network that is very similar both in volume and in organization, but in autism this network is much more loosely connected. This functional underconnectivity was observed in the anterior-posterior connections. The results expand the theory of cortical underconnectivity in autism to the resting state of the brain. NeuroReport 17:1687-1690 (C) 2006 Lippincott Williams \& Wilkins.

Keywords: autism, corpus callosum, functional connectivity, functional magnetic resonance imaging (fMRI), resting-state network

\section{Introduction}

Converging evidence from functional magnetic resonance imaging (fMRI) studies has indicated that autism is a system-wide disorder with reduced synchronization of the time course of brain activation found in several tasks, such as language processing [1], working memory [2], executive functioning [3], and visual imagery [4]. All these previous fMRI studies explored brain activation during task performance. Fixation on the 'resting state' in fMRI tasks provides a new domain to measure cortical synchronization. The flow of mental events continues even during the resting state, activating a set of brain regions that have been associated with a 'default mode' of brain function [5]. The brain regions identified as default mode areas have been localized most often to medial structures including the medial frontal gyrus, the anterior cingulate, the posterior cingulate, and the precuneus (PREC) [5-8].

Researchers have found that individuals with autism report very different internal thoughts than control participants $[9,10]$. The main objective of the present study was to investigate the brain activation and synchronization in autism in the most 'diluted', content-free baseline 'resting state'. Resting-state fMRI was used in this study to investigate both the volume and location of activation, and functional connectivity in people with autism and control participants. Examining functional connectivity during a baseline resting state provides an opportunity to pool data across several studies, resulting in an unusually large sample size for a functional imaging analysis. To our knowledge, this is the first study to investigate functional connectivity during rest in autism. In addition, we examine the relationship between functional connectivity during resting baseline and the size of the corpus callosum, a major component of anatomical connectivity.

On the basis of previous findings of regions activated during rest in the typical population, and brain activation findings in autism during task performance, we set out to examine the following hypotheses. First, we predicted that people with autism would show reduced functional connectivity relative to controls during the resting state. The rationale behind this hypothesis is that inward thinking during rest involves subprocesses, such as representation, monitoring, evaluation, and integration. These processes take effect by the coordinated interaction of the medial cortical structures [11], which demands robust connectivity. Second, we anticipated that functional connectivity in autism would be positively correlated with the corpus callosum size. This hypothesis is based on our findings of such correlation in autism during task performance. In general, autism may affect all types of brain activity, and the resting state may be no exception.

\section{Method}

Participants

Fifty-seven high-functioning individuals with autism and 57 control participants were included in the analyses. Participants were matched on the basis of age (autism mean $\pm S D$ : 
$24 \pm 10.6$ years; control mean \pm SD: $24 \pm 9.0$ years), full scale intelligence quotient (autism: $106 \pm 16.2$; control: $113 \pm 11.3$ ), and verbal intelligence quotient (autism: $104 \pm 16.1$; control: $108 \pm 10.5$ ). Four participants in the autism group and five in the control group were women; seven participants in the autism group and four control participants were lefthanded. The diagnosis of autism was established using the Autism Diagnostic Interview-Revised [12] and the Autism Diagnostic Observation Schedule-Generic [13]. The detailed description of participant selection and exclusion is reported elsewhere [3]. Each participant signed an informed consent that had been approved by the University of Pittsburgh and Carnegie Mellon University Institutional Review Boards.

\section{Experimental paradigm}

The resting-state network was analyzed using data from six separate fMRI autism studies performed at the Center for Cognitive Brain Imaging (Carnegie Mellon University). The studies addressed executive function (Tower of London task), various language-related tasks (visual imagery, irony comprehension, understanding discourse), response inhibition, and 'theory of mind'.

In addition to the two or three conditions of the cognitive task, each of these studies had a separate fixation task, when an asterisk was presented at the middle of the computer screen and the participant was instructed to focus on it, think about nothing and 'clear your mind'. Each study had four or five blocks of fixations distributed throughout the experiment. Each block lasted for $24 \mathrm{~s}$. Therefore, it was possible to perform the analysis of brain activation during fixation for a large pool of participants (57 in each group). Sixteen participants with autism and 15 control participants took part in more than one study.

\section{Functional magnetic resonance imaging parameters}

For all the studies but the Tower of London, the imaging was carried out on a $3 \mathrm{~T}$ Siemens Allegra scanner (Siemens, Erlangen, Germany). The details of the stimulus presentation and data acquisition parameters are reported elsewhere [4]. Functional scans were performed at $\mathrm{TR}=1000 \mathrm{~ms}$, with 16 oblique-axial 5-mm slices, $1-\mathrm{mm}$ slice gap, and an in-plane resolution of $3.125 \times 3.125 \mathrm{~mm}^{2}$. For the Tower of London study, imaging was performed on GE $1.5 \mathrm{~T}$ and $3 \mathrm{~T}$ scanners (General Electric, Milwaukee, Wisconsin, USA) with similar parameters except for $\mathrm{TR}=3000 \mathrm{~ms}$ and 14 slices. In addition to functional scans, T1-weighted scans were also obtained.

\section{Distribution of activation}

The data were analyzed using SPM99 (Wellcome Department of Cognitive Neurology, London, UK). Images were corrected for slice timing and motion, normalized to the Montreal Neurological Institute template, resampled to $2 \times 2 \times 2 \mathrm{~mm}^{3}$ voxels, and smoothed with an $8-\mathrm{mm}$ Gaussian kernel. For each participant, a contrast between the fixation and all cognitive tasks was computed, and the group analyses were performed using a random-effects model.

\section{Functional connectivity}

Functional connectivity was computed for each participant and each pair of regions of interest (ROIs) as a correlation between the average time courses of all the voxels of the first and second ROIs. Twelve functional ROIs (spheres with a radius of $6 \mathrm{~mm}$ ) were defined that best captured the peaks of activation in the combined group activation map. Labels for these 12 ROIs [the posterior cingulate cortex (PCC), ventral anterior cingulate cortex (VACC), PREC, paracentral lobule (PCL), plus four bilateral ROIs, namely medial/ middle prefrontal cortex (MPFC), inferior parietal cortex (IPC), parahippocampal gyrus (PHG), and inferolateral temporal cortex (insula)] were assigned with reference to the parcellation of the Montreal Neurological Institute template subject [14]. The averaged time courses were high-pass filtered (cut-off value range 120-225s) and had the linear trend removed. The correlation was computed on the images belonging only to the fixation condition, so it reflects the interaction between the activation in two areas when the participant is at rest. The first $6 \mathrm{~s}$ of each fixation block were removed to accommodate the hemodynamic delay. Fisher's $r$ to $z^{\prime}$ transformation was applied to the correlation coefficients for each participant. For the individuals who participated in multiple studies (i.e. had multiple scans), the appropriate connectivities were averaged. We also performed the analysis using a single random scan for these participants, and obtained very similar results. In order to summarize the connectivity differences, the 12 ROIs were grouped into larger regions in separate analyses on the basis of lobes, anterior or posterior locations, and hemispheres. Group differences in connectivity were computed using a single $z$-value (averaged across the ROI grouping) for each participant.

\section{Corpus callosum morphometry}

The cross-sectional area of the midsagittal slice of the corpus callosum was measured using the parcellation scheme described by Witelson [15] (see [4] for details).

\section{Limitations}

As this study used fixation blocks in experiments, the nature of the data limits our capability to estimate very lowfrequency components of interactions between the brain areas (the fixation block duration was $24 \mathrm{~s}$ ). There is also a possibility of some aftereffects of specific tasks on the baseline activity, although all efforts were made to eliminate such effects. No reason, however, exists to assume that such effects might affect the two groups differently.

\section{Results}

\section{Functional connectivity}

Despite similar levels of activation in autism and control groups, the functional connectivity between many ROIs was lower for the participants with autism. This effect was widespread, such that out of a total 66 pairwise connectivities computed for the set of 12 ROIs, 94\% showed lower connectivity for the participants with autism. To characterize these differences statistically, ROIs were grouped together based on a few principles.

First, the connections of the lobe-based groups of ROIs (frontal with other lobes) were compared for the two groups. The reason for this grouping is that the most affected functional connectivity in autism is between frontal areas and other cortical areas $[1,3,4]$. To compute this functional connectivity, all connectivities of the ROI pairs consisting of any frontal (VACC, left MPFC, right MPFC) and any parietal (PCC, PREC, left IPC, right IPC) ROIs were 
averaged, that is, we averaged the connectivities of VACC with PCC, VACC with PREC, etc. Frontal-parietal connectivity was lower for the autism group, but the difference did not reach significance (autism mean: 0.30, control mean: 0.36 , NS). At the same time, the connectivity of the anterior cingulate region (VACC) with the posterior cingulate and precuneus group (PCC + PREC) was significantly lower for the autism group (autism mean: 0.27, control mean: 0.37, two-tail $t$-test; $t(112)=2.05, P<0.05)$.

Second, the connections of the left and right parahippocampal areas with the rest of the ROIs were explored separately. The connectivity of the left PHG with the rest of the ROIs was significantly lower for the autism group (autism mean: 0.14 , control mean: $0.23 ; t(112)=2.44, P<0.05$ ), but this was not the case for the right PHG (autism mean: 0.16 , control mean: 0.22 , NS).

Additionally, the 12 ROIs were grouped on the basis of hemisphere, resulting in three networks (within left hemisphere, within right hemisphere, and between hemisphere connections). Medial ROIs (VACC, PCL, PCC, and PREC) were excluded from this analysis. Within left hemisphere connectivity was significantly lower for the group with autism (autism mean: 0.16, control mean: 0.24; $t(112)=2.05$, $P<0.05)$. No reliable differences were seen in within right hemisphere connectivity (autism mean: 0.17, control mean: 0.22 , NS) or between hemispheres connectivity (autism mean: 0.23 , control mean: $0.30, \mathrm{NS}$ ).

\section{Corpus callosum size and functional connectivity}

The size of the entire corpus callosum, as well as some subregions, was correlated with the functional connectivity measures in both groups. The size of the rostral body, an anterior subregion of the corpus callosum, was reliably positively correlated with the functional connectivity between left frontal MPFC and posterior PCC regions in the autism group $[r=0.36, t(55)=2.84, P<0.01]$, whereas the control group showed no such correlation $(r=-0.07, \mathrm{NS})$. The scatter plots for these data are presented in Fig. 1. The same corpus callosum region was also reliably positively correlated with the functional connectivity between left and right parahippocampal areas in the autism group $[r=0.29$,

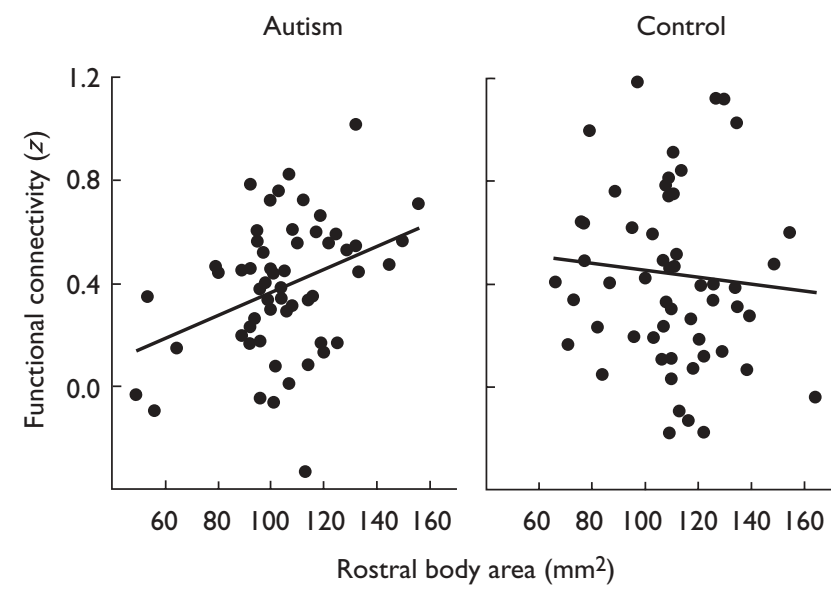

Fig. I Correlations between functional connectivity and the corpus callosum size. The connectivity of left frontal medial/middle prefrontal cortex and posterior cingulate cortex regions vs. the size of rostral body of corpus callosum: autism, $r=0.36$; control, $r=-0.07$. $t(55)=2.25, P<0.05]$, whereas the control group showed no such correlation $(r=-0.07, \mathrm{NS})$.

The result of positive correlation between the size of the anterior areas of the corpus callosum and the functional connectivity of anterior-posterior connections in autism is similar to the findings in our previous studies [3,4] related to functional connectivity during the performance of cognitive tasks. These findings provide insight concerning an anatomical basis of underconnectivity in autism.

\section{Group similarity in brain activation}

The resting-state brain activation for the group with autism is presented in Fig. 2a. The activation was very similar for both groups. Direct comparison of the two groups' activation showed no differences at $P=0.001$, extent threshold of 100 voxels; when the extent threshold was lowered to 20 voxels, participants with autism activated more in two left hemisphere areas: middle/superior frontal gyrus (26 voxels) and supramarginal gyrus (37 voxels). Control participants showed higher activation in the right middle temporal gyrus (37 voxels) (Fig. $2 b$ ). We concluded that the differences in activation between the two groups were very small. The following areas were involved in the resting-state network for both groups: PCC, VACC, PREC, PCL, and bilaterally, MPFC, IPC, PHG, and inferolateral temporal cortex.

(a)

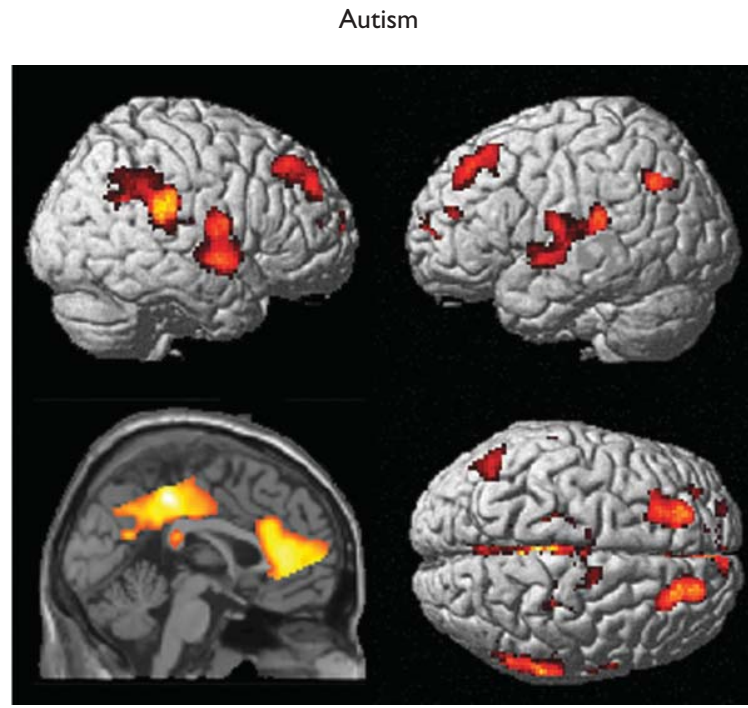

(b)

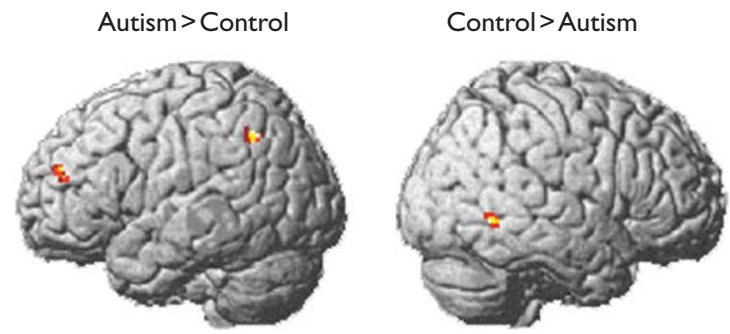

Fig. 2 Resting-state network activation. (a) Autism group resting-state activation $(P<0.00 \mathrm{l})$. (b) Minor group differences in the resting-state activation: autism group $>$ control group (left), control group $>$ autism group (right). Other brain surface renderings (not shown) contain no differences $(P<0.001)$. 


\section{Discussion}

Previously, we had postulated the cortical underconnectivity theory of autism [1] on the basis of brain activity characteristics in a wide range of cognitive tasks. The present study extends the underconnectivity theory to the domain of a task-free, resting state. The central finding of this study was that the participants with autism showed underconnectivity between anterior and posterior regions that activate in fixation. The medial structures, anterior cingulate and posterior cingulate were functionally underconnected in autism relative to control participants. These findings reveal that functional underconnectivity in autism is not restricted to complex tasks, but is also prevalent during rest, so the lack of coordination among neural centers in autism is pervasive.

Lower functional connectivity between anterior and posterior medial cortex is interesting considering the possible role of these resting state (default mode) centers. A number of studies indicate that both anterior medial cortex (medial frontal gyrus and/or anterior cingulate cortex) and posterior medial cortex (PCC and/or PREC) are involved in selfreferential processing [16,17]. Studies have also reported impairments in self-consciousness in autism [18]. It is possible that people with autism might not be generating spontaneous and self-related thought processes during fixation. It is also possible that these regions are involved in emotional processing through the connections between VACC and PCC [17]. The link between PCC and VACC is believed by some to be important in connecting higher cortical (conscious) processing and more basic (possibly unconscious) processing related to autonomic state [8].

A second major result pertains to the correlation between fixation functional connectivity in autism and the corpus callosum size. fMRI studies during task performance have found such correlation previously [3,4]. It is interesting that this effect prevails even when participants are not doing a cognitive task. This indicates that whatever factor is depressing the functional connectivity in autism during task performance is also operative during a resting state. Finally, we found that the resting-state network activation was similar in both groups, unlike a previous study that found reduced activation in autism in the default mode network regions [19]. The source of this difference might be related to the task they used (the counting Stroop task) that produces activation related to inhibition, which is seen in medial cingulate regions.

\section{Conclusions}

These results provide new converging evidence for the hypothesis that autism is a distributed neural systems disorder rather than a focal disorder and that the disorder is manifest even when people are not performing any particular task.

\section{References}

1. Just MA, Cherkassky VL, Keller TA, Minshew NJ. Cortical activation and synchronization during sentence comprehension in highfunctioning autism: evidence of underconnectivity. Brain 2004; 127: 1811-1821.

2. Koshino H, Carpenter PA, Minshew NJ, Cherkassky VL, Keller TA, Just MA. Functional connectivity in an fMRI working memory task in high-functioning autism. Neuroimage 2005; 24:810-821.

3. Just MA, Cherkassky VL, Keller TA, Kana RK, Minshew NJ. Functional and anatomical cortical underconnectivity in autism: evidence from an fMRI study of an executive function task and corpus callosum morphometry. Cereb Cortex 2006.

4. Kana RK, Keller TA, Cherkassky VL, Minshew NJ, Just MA. Sentence comprehension in autism: thinking in pictures with decreased functional connectivity. Brain 2006; 129:2484-2493.

5. Raichle ME, MacLeod AM, Snyder AZ, Powers WJ, Gusnard DA, Shulman GL. A default mode of brain function. Proc Natl Acad Sci 2001; 98:676-682.

6. Shulman GL, Fiez JA, Corbetta M, Buckner RL, Meizin FM, Raichle ME, Petersen SE. Common blood flow changes across visual tasks: II. decreases in cerebral cortex. J Cogn Neurosci 1997; 9: 648-663.

7. Gusnard DA, Raichle ME. Searching for a baseline: functional imaging and the resting human brain. Nat Rev Neurosci 2001; 2:684-694.

8. Greicius MD, Krasnow B, Reiss AL, Menon V. Functional connectivity in the resting brain: a network analysis of the default mode hypothesis. Proc Natl Acad Sci 2003; 100:253-258.

9. Hurlburt R, Happé F, Frith U. Sampling the form of inner experience in three adults with asperger syndrome. Psychol Med 1994; 24: 385-395.

10. Frith U, Happé F. Theory of mind and self-consciousness: what is it like to be autistic? Mind Lang 1999; 14:1-22.

11. Northoff G, Bermpohl F. Cortical midline structures and the self. Trends Cogn Sci 2004; 8:3.

12. Lord C, Rutter M, LeCouteur A. Autism diagnostic interview-revised: a revised version of a diagnostic interview for caregivers of individuals with possible pervasive developmental disorders. J Autism Dev Disord 1994; 24:659-685.

13. Lord C, Risi S, Lambrecht L, Cook EH Jr, Leventhal BL, DiLavore PC, et al. The autism diagnostic observation schedule-generic: a standard measure of social and communication deficits associated with the spectrum of autism. J Autism Dev Disord 2000; 30:205-223.

14. Tzourio-Mazoyer N, Landeau B, Papathanassiou D, Crivello F, Etard O, Delcroix $\mathrm{N}$, et al. Automated anatomical labeling of activations in SPM using a macroscopic anatomical parcellation of the MNI MRI singlesubject brain. Neuroimage 2002; 15:273-289.

15. Witelson SF. Hand and sex differences in the isthmus and genu of the human corpus callosum: a postmortem morphological study. Brain 1989; 112:799-835.

16. Ochsner KN, Beer JS, Robertson ER, Cooper JC, Gabrieli JDE, Kihlstrom JF, D'Esposito M. The neural correlates of direct and reflected self-knowledge. Neuroimage 2005; 28:797-814.

17. Vogt BA, Laureys S. Posterior cingulate, precuneal and retrosplenial cortices: cytology and components of the neural network correlates of consciousness. In: Laureys S, editor. Progress in brain research. Vol 150. Amsterdam: Elsevier; 2005. pp. 205-217.

18. Dawson G, McKissick FC. Self-recognition in autistic children. J Autism Dev Disord 1984; 14:383-394.

19. Kennedy DP, Redcay E, Courchesne E. Failing to deactivate: resting functional abnormalities in autism. Proc Natl Acad Sci 2006; 103: 8275-8280. 